Somnologie 2015 $\cdot 19: 144$

DOI 10.1007/s11818-015-0022-8

Online publiziert: 27. August 2015

(c) Springer-Verlag Berlin Heidelberg 2015

Geert Mayer

Hephata Klinik Schwalmstadt, Schwalmstadt, Deutschland

\title{
Qualitätssicherung in der Schlafmedizin
}

zinischer Fachgesellschaften (AWMF) initiiert. Man könnte das auch die zwei Seiten einer Medaille nennen. Im Prinzip geht es darum, überflüssige Prozeduren im Patient-Arzt-Dialog zu vermeiden.

Auch in der Schlafmedizin sind wir vielen formalen Abläufen unterworfen, die wir einhalten müssen, egal ob sie Sinn machen oder nicht. Ohne Dokumentation der Abläufe kein Geld. $\mathrm{Ob}$ Regeln wie die Bewertung medizinischer Untersuchungs- und Behandlungsmethoden gem. $₫ 135$ Abs. 1 des Fünften Sozialgesetzbuches (BUB-Bestimmungen) inhaltlich und wirtschaftlich Sinn machen, ist eine Fragestellung, der wir in Hessen nachgegangen sind (Mayer et al., in diesem Heft). Wenn zur Diagnostik eindeutiger klinischer Fälle im Vorfeld diagnostische Methoden eingesetzt werden, die überflüssig sind, weil sie nicht spezifisch genug sind, macht das wenig Sinn. Wenn allerdings große Bevölkerungsgruppen auf schlafbezogene Atmungsstörungen gescreent werden sollen, kann das Sinn machen. Ein anderer Aspekt, der kaum eine Rolle spielt in der jetzigen Form der Qualitätssicherung, ist das große Vertrauen in die Technik. Im Schlaf generieren wir inzwischen eine Fülle von Daten, die wir in ihrer Qualität nicht überprüfen, weil wir uns darauf verlassen, dass die Technik so optimiert wurde, dass sie unsere Fragestellungen beantwortet. Gerade bei den neurophysiologischen Parametern ist es aber immens wichtig, die Art der abgeleiteten Graphoelemente abhängig von der Einstellung der Geräte zu lesen. Dies kann man im Prinzip nur wissen, wenn man einmal selbst die Einstellungen einzelner Kanäle verändert hat und sich die Auswirkungen auf ihr Erscheinungsbild angesehen hat. Diesen
Prozess zu erfassen, hat sich das Somnonetz zur Aufgabe gemacht (Siewert et al., in diesem Heft). Es dient seit 2014 der optimierten Erfassung der Daten zur Qualitätssicherung im Schlaflabor auf elektronischem Wege. Hier sind sicher in Zukunft noch viele Optimierungsprozesse nötig, um tatsächlich Qualität zu erfassen. Der Sprung in die Vergangenheit des Entstehungsprozesses des ersten zeitgeberfreien Labors kann den Einfluss der Technik auf unseren Erkenntnisprozess eindrucksvoll dokumentieren. Beim Blickin die Zukunft heißt dies, Mut zu haben, neue Techniken zu erproben und sie uns nicht überstülpen zu lassen. In diesem Sinne sollte jeder Schlafmediziner über die Anwendung neuer Technologien für Diagnostik und Therapie nachdenken und Vorschläge einbringen für bessere, einfache Anwendungen. Die Fachgesellschaft wird sich, wenn die Methode effektiv ist, um den Erlös kümmern.

\section{Korrespondenzadresse

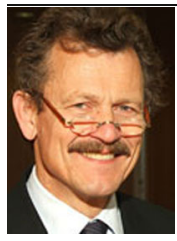 \\ Prof. Dr. G. Mayer \\ Hephata Klinik Schwalmstadt Schimmelpfengstr. 6, 34613 Schwalmstadt, Deutschland geert.mayer@hephata.com}

Interessenkonflikt. G. Mayer gibt an, dass kein Interessenkonflikt besteht. 